\title{
Distinguishing number and adjacency properties
}

\author{
Anthony Bonato* and Dejan Delić* \\ Dedicated to the memories of Roland Fraïssé and Michael O. Albertson
}

\begin{abstract}
The distinguishing number of countably infinite graphs and relational structures satisfying a simple adjacency property is shown to be 2 . This result generalizes both a result of Imrich et al. on the distinguishing number of the infinite random graph, and a result of Laflamme et al. on homogeneous relational structures whose age satisfies the free amalgamation property.

AMS 2000 SUBJECT CLASSificAtions: Primary 05C75, 05C75; secondary $05 \mathrm{C} 25$.

KEYWORDS AND PHRASES: Distinguishing number, adjacency property, relational structure, infinite random graph, homogeneous structure.
\end{abstract}

\section{Introduction}

One of the most widely studied infinite graphs is the Rado or infinite random graph, written $R$. A graph satisfies the existentially closed or e.c. adjacency property if for all finite disjoint sets of vertices $A$ and $B$ (one of which may be empty), there is a vertex $z \notin A \cup B$ joined to all of $A$ and to no vertex of $B$. By a back-and-forth argument, $R$ is the unique isomorphism type of countably infinite graphs that is e.c. Further, $R$ is homogeneous: every isomorphism between finite induced subgraphs extends to an automorphism. For a survey of these and other results on $R$, see [3].

The distinguishing number of a graph $G$, written $D(G)$, is the least positive integer $n$ such that there exists an $n$-colouring of $V(G)$ (not necessarily proper) so that no non-trivial automorphism preserves the colours. Rigid graphs (which possess no non-trivial automorphisms) have distinguishing number 1, and $D(G)$ may be viewed as the minimum number of colours needed to make $G$ rigid. The parameter $D(G)$ was introduced by Albertson and Collins [1].

The distinguishing number of graphs generalizes in a straightforward fashion to relational structures. A relation on a set $X$ is a set of $n$-tuples from

\footnotetext{
${ }^{*}$ The authors gratefully acknowledge support from NSERC and MITACS.
} 
$X$, where $n>0$ is its arity. A signature $\mu$ is a (possibly infinite) sequence $\left(\mu_{i}: i \in I\right)$ of positive integers. A relational structure $S$ with signature $\mu$ consists of a non-empty vertex set $V(S)$, and a set of relations $R_{i}$ on $V(S)$ for $i \in I$ of arity $\mu_{i}$. Isomorphisms, induced subgraphs, distinguishing number, and many other notions from graph theory generalize naturally to relational structures. For background on relational structures, we refer the reader to [4]. All graphs we consider are simple.

While most research on the distinguishing number has focused on the finite case, recent work considers infinite structures as well. Imrich, Klavžar, and Trofimov [5] recently proved (among other things) that $D(R)=2$. Laflamme, Nguyen Van Thé, and Sauer [7] generalized this fact by showing that a homogeneous relational structure with minimal arity 2, whose age (that is, set of isomorphism types of induced finite substructures) satisfies the free amalgamation property has distinguishing number 2 . In [8], $D(T)$ is determined for infinite, locally finite trees $T$.

In this short note, we introduce an adjacency property called weak-e.c. for countable relational structures (generalizing the e.c. property) which is a sufficient condition to have distinguishing number at most 2 ; see Theorem 1.2. As a consequence of this fact, in Corollary 1.4 we show that homogeneous structures whose age has free amalgamation have distinguishing number 2. Our results generalize the results of $[5 ; 7]$ stated in the previous paragraph. Further, they supply a large class of relational structures with distinguishing number 2. For example, there are $2^{\aleph_{0}}$ many non-isomorphic countable graphs with the weak-e.c. property; see [2].

Definition 1.1. A graph $G$ that is not a clique is weak-e.c. if for each pair $u, v$ of (possibly equal) non-joined vertices and a finite set $T$ of vertices containing neither $u$ nor $v$, there is a vertex $z$ joined to $u$ and $v$ but not joined nor equal to a vertex in $T$.

The graph $R$ has the weak-e.c. property, as does the universal homogeneous triangle-free graph, although the latter graph is not e.c. Note that the weak-e.c. property implies that the graph has diameter 2 , and has no vertex of finite degree.

If $S$ is a relational structure, then the (Gaifman) graph of $S$, written $G(S)$, has vertices those of $S$ with two vertices $x$ and $y$ joined if $x \neq y$ and only if they appear together in some tuple in a relation of $S$. Note that an automorphism of $S$ induces an automorphism of $G(S)$.

A relational structure $S$ is weak-e.c. if $G(S)$ is weak-e.c. Our main result is the following. 


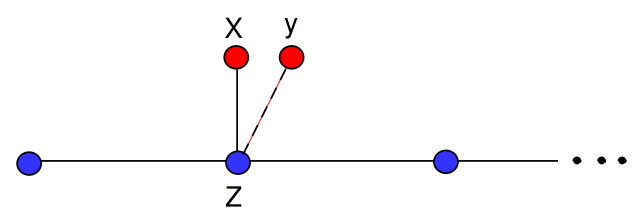

Figure 1: Fixing $x$ and $y$ in Claim 1.

Theorem 1.2. If the countable relational structure $S$ satisfies the weak-e.c. property, then $D(S) \leq 2$.

Proof. Let $G=G(S)$. We prove first that $D(G(S)) \leq 2$. We actually prove that a weak-e.c. graph satisfies another adjacency property, which in turn implies a distinguishing number at most 2. A graph $G$ satisfies (\$) if there is an induced ray $Z$ (that is, an infinite one-way path) in $G$ such that for all pairs of distinct vertices $x$ and $y$ not in $Z$, there is a vertex in $Z$ joined to exactly one of either $x$ or $y$.

Claim 1. Property (\$) implies that $D(G) \leq 2$.

To see this, let $\mathbf{B}$ - the blue vertices - be the vertices of the induced ray $Z$, and let $\mathbf{R}$, the red vertices, be the vertices in $V(G) \backslash \mathbf{B}$. It is straightforward to see that no automorphism $f$ of $G$ preserving the colour sets can move an element of $\mathbf{B}$. We claim that $f$ restricted to $\mathbf{R}$ is the identity. To see this, let us suppose that $f(x)=y$ for some distinct red vertices $x$ and $y$. By ( $\mathbf{s})$, there is a blue vertex $z$ joined to (say) $x$ but not $y$. See Figure 1. But this is a contradiction as $f$ fixes $z$. The proof of the Claim 1 follows.

The fact that $D(G) \leq 2$ is implied by the following claim.

Claim 2. The weak-e.c. property implies (\$).

For the proof of Claim 2, enumerate all unordered pairs of distinct vertices of $G$ as

$$
R_{-1}=\left\{\left\{x_{i}, y_{i}\right\}: i \in \mathbb{N}\right\}
$$

We inductively process pairs of vertices from $R_{-1}$. Each pair will be labeled processed or unprocessed; at the beginning of the base step, all pairs are unprocessed.

By the weak-e.c. property, there is a vertex $z_{0}$ joined to $x_{0}$ that is neither joined nor equal to $y_{0}$ (in the notation of the definition of weak-e.c., we are setting $u=v=x_{0}$, and $\left.T=\left\{y_{0}\right\}\right)$. Delete all pairs $\left\{x_{j}, y_{j}\right\}$ from $R_{-1}$ 
containing $z_{0}$ to form the set of pairs $R_{0}$. Label $\left\{x_{0}, y_{0}\right\}$ as processed. For ease of notation, we relabel the remaining pairs of $R_{-1}$ so that

$$
R_{0}=\left\{\left\{x_{i}, y_{i}\right\}: i \in \mathbb{N}\right\}
$$

For $n \geq 0$ fixed, assume that we have found a finite set of distinct vertices $Z_{n}$ and a set $R_{n}$ of pairs from $V(G)$ with the following properties. For simplicity, we assume the pairs of $R_{n}$ have been relabeled so that

$$
R_{n}=\left\{\left\{x_{i}, y_{i}\right\}: i \in \mathbb{N}\right\}
$$

For each $R_{n}$ and $k \geq 0$, define its $k$-initial segment $R_{n}[k]$ to consist of the set

$$
\left\{\left\{x_{0}, y_{0}\right\},\left\{x_{1}, y_{1}\right\}, \ldots,\left\{x_{k}, y_{k}\right\}\right\} .
$$

We require that $R_{n+1}[n]=R_{n}[n]$. Indices of the $x_{i}$ and $y_{i}$ in (1) to (3) below refer to the enumeration of pairs in $R_{n}$.

1. For each $0 \leq i \leq n$, there is a vertex $z_{i} \in Z_{n}$ that is distinct from $x_{i}$ and $y_{i}$, and is joined to exactly one of $x_{i}$ or $y_{i}$. The vertex $z_{i}$ is not equal to any $x_{j}$ nor $y_{j}$, where $0 \leq j \leq i-1$.

2. The set $Z_{n}$ induces in $G$ an $n$-path with terminal vertices $z_{0}$ and $z_{n}$.

3. For all $z \in Z_{n}$, the vertex $z$ is not in a pair in $R_{n}$. Each of the pairs $\left\{x_{i}, y_{i}\right\}$, where $0 \leq i \leq n$, are labeled as processed.

To complete the inductive step, we note that the vertex $z_{n}$ may or may not be joined to the vertices $x_{n+1}$ or $y_{n+1}$. We do know for certain that $z_{n}$ is not equal to either $x_{n+1}$ or $y_{n+1}$ by item (3) of the induction hypothesis. By the weak-e.c. property, we may find a vertex $z^{\prime}$ joined to $z_{n}$ but not joined nor equal to any vertex in

$$
T^{\prime \prime}=\left(Z_{n} \backslash\left\{z_{n}\right\}\right) \cup\left\{x_{0}, \ldots, x_{n+1}\right\} \cup\left\{y_{0}, \ldots, y_{n+1}\right\}
$$

The vertex $z^{\prime}$ will not be our choice for $z_{n+1}$, but plays an intermediary role in finding such a vertex. Define $T^{\prime}$ to be the set of vertices in $\left\{x_{0}, \ldots, x_{n}\right\} \cup$ $\left\{y_{0}, \ldots, y_{n}\right\}$ not equal to either $x_{n+1}$ or $y_{n+1}$. (We note that since we are enumerating unordered pairs in $R_{n}$, either of the vertices $x_{n+1}$ or $y_{n+1}$ may be equal to some $x_{i}$ or $y_{i}$ for some $1 \leq i \leq n$.) By the weak e.c. property with $u=z^{\prime}$ and $v=x_{n+1}$, there is a vertex $z_{n+1}$ joined to $z^{\prime}$ and $x_{n+1}$, but not joined nor equal to a vertex in

$$
T=Z_{n} \cup T^{\prime} \cup\left\{y_{n+1}\right\} .
$$


In particular, $z_{n+1}$ is distinct from, and joined to exactly one of $x_{n+1}$ or $y_{n+1}$ as required in item (1). Set $Z_{n+1}=Z_{n} \cup\left\{z^{\prime}, z_{n+1}\right\}$, and note that the subgraph induced by $Z_{n+1}$ is a path with terminal vertices $z_{0}$ and $z_{n+1}$. Form $R_{n+1}$ by deleting any pairs in $R_{n}$ containing $z^{\prime}$ or $z_{n+1}$, and then relabeling the pairs so that $R_{n+1}=\left\{\left\{x_{i}, y_{i}\right\}: i \in \mathbb{N}\right\}$. Note that this deletion preserves the property that $R_{n+1}[n]=R_{n}[n]$, since $\left\{x_{n+1}, y_{n+1}\right\}$ will not be deleted as $z_{n+1}$ and $z^{\prime}$ were chosen to be distinct from these two vertices. Hence, properties (1), (2), and (3) are satisfied with this choice of $z_{n+1}, R_{n+1}$, and $Z_{n+1}$.

Set

$$
Z=\bigcup_{n \in \mathbb{N}} Z_{n}
$$

and let $P$ be the vertices in $V(G) \backslash Z$. The subgraph induced by $Z$ is a ray. Note that each distinct pair of vertices $\{x, y\}$ in $P$ is processed in the above induction as some pair $\left\{x_{i}, y_{i}\right\}$. In particular, there is a vertex in $Z$ joined to exactly one of $x$ or $y$. Hence, Claim 2 follows.

Now, let $\operatorname{Aut}(S, \mathbf{B}, \mathbf{R})$ be the automorphism group of the relational structure $S$ with two additional unary predicates, $\mathbf{B}$ and $\mathbf{R}$, identified with the colour sets $\mathbf{B}$ and $\mathbf{R}$, respectively. The property that $D(X) \leq 2$ is equivalent to $\operatorname{Aut}(S, \mathbf{B}, \mathbf{R})$ being the trivial group. The proof now follows from Claims 1 and 2 since $\operatorname{Aut}(S, \mathbf{B}, \mathbf{R})$ is isomorphic to a subgroup of $\operatorname{Aut}(G(S), \mathbf{B}, \mathbf{R})$.

Theorem 3.1 of Imrich et al. [5] follows directly from Theorem 1.2 as a corollary, since $R$ is weak-e.c. We point out that the property (\$) introduced in Theorem 1.2 is a more general sufficient condition for having distinguishing number at most 2 than the weak-e.c. property. For example, the infinite random bipartite graph $R_{B}$ satisfies ( $)$ and hence, has distinguishing number 2 by Claim 1 in the proof of Theorem 1.2, but is not weak-e.c. since its diameter is not 2. (The proof that $R_{B}$ satisfies ( $)$ is similar to the proof of Claim 2, and so is omitted. The additional detail in the inductive step is to consider cases of the colours of $x_{n+1}$ and $y_{n+1}$.)

The high degree of symmetry exhibited by $R$ may be formalized in a notion which applies to many other relational structures. A structure is homogeneous if each isomorphism between finite induced substructures extends to an automorphism. Fix $\mathcal{K}$ a class of structures of the same signature that is closed under isomorphisms. An amalgam is a 5-tuple $(A, f, B, g, C)$ such that $A, B$, and $C$ are structures in $\mathcal{K}$, and $f: A \rightarrow B, g: A \rightarrow C$ are embeddings (that is, isomorphisms onto their images). Then $\mathcal{K}$ has the amalgamation property, written $(A P)$, if for every amalgam $(A, f, B, g, C)$, 
there exist both a structure $D \in \mathcal{K}$ and embeddings $f^{\prime}: B \rightarrow D, g^{\prime}: C \rightarrow D$ such that $f^{\prime} \circ f=g^{\prime} \circ g$. The connection between classes with (AP) and homogeneous structures is made transparent by Fraïssé's theorem, which we restate as Theorem 1.3 below. A structure $G$ is universal in $\mathcal{K}$ if each member $\mathcal{K}$ is isomorphic to an induced substructure of $G$. The class $\mathcal{K}$ has the joint embedding property or (JEP) if for every pair $B$ and $C$ in $\mathcal{K}$, there is a $D \in \mathcal{K}$ so that $B$ and $C$ are isomorphic to induced substructures of $D$. (If we allow empty structures, then (JEP) is a special case of (AP). Since we only consider non-empty structures, we will not use this convention.)

Theorem 1.3 (Fraïssé, [4]). Let $\mathcal{K}$ be a class of finite structures with the same signature closed under isomorphisms. Then the following are equivalent.

1. The class $\mathcal{K}$ has (AP), (JEP), and is closed under taking induced substructures.

2. There is a countable universal and homogeneous structure $S$ whose age is $\mathcal{K}$, and which is a limit of a chain of structures from $\mathcal{K}$.

The structure $S$ in Theorem 1.3 (2) is called the Fraïssé limit of $\mathcal{K}$. For example, $R$ is the Fraïssé limit of the class finite graphs. Note that $S$ has the following useful property. Suppose that $A, B$ are structures in the age of $S$, with $A$ an induced substructure of both $B$ and $S$. Then there is an isomorphism $\beta$ from $B$ to an induced substructure of $S$ so that $\beta$ is the identity on $A$. We say that $B$ amalgamates into $S$ over $A$.

Given relational structures $S_{1}$ and $S_{2}$ of the same signature their union (or free amalgam) $S_{1} \cup S_{2}$ has vertices the union of the vertex sets of $S_{1}$ and $S_{2}$, and whose relations are the union of the relations of $S_{1}$ and $S_{2}$. Note that $S_{1}$ and $S_{2}$ may not in general have disjoint vertex sets; in which case we say that the union $S_{1} \cup S_{2}$ is formed with intersection $V\left(S_{1}\right) \cap V\left(S_{2}\right)$. If $V\left(S_{1}\right) \cap V\left(S_{2}\right)$ is empty, then $S_{1} \cup S_{2}$ is simply their disjoint union. A class of finite relational structures with fixed signature so that $\mathcal{K}$ closed under isomorphism has free amalgamation if it is closed under taking unions of structures; that is, if $S_{1}$ and $S_{2}$ are in $\mathcal{K}$, then $S_{1} \cup S_{2} \in \mathcal{K}$.

The following corollary gives a short and elementary proof of Theorem 3.1 of LaFlamme et al. [7]. To avoid degenerate cases in the following theorem, we only consider non-null structures; that is, structures $S$ where $G(S)$ contains edges.

Corollary 1.4. Let $S$ be a countable, homogeneous, non-null structure with minimal arity of at least two whose age has free amalgamation. Then $D(S)=2$. 


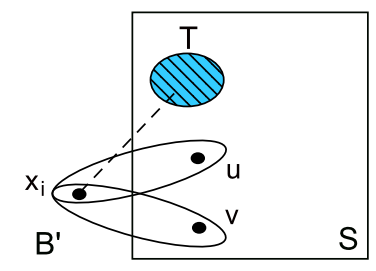

Figure 2: Amalgamating $B^{\prime}$ into $S$ over $A$.

Proof. We first show that $S$ satisfies the weak-e.c. property. We may then apply Theorem 1.2 to prove that $D(S) \leq 2$. By homogeneity, $S$ is not rigid so $D(S)=2$.

Now fix $u, v$ and a finite set $T$ in $V(S)$ so that $u, v$ are not joined in $G(S)$, and $u, v \notin T$. Fix a $k$-tuple $\bar{x}=\left(x_{1}, \ldots, x_{k}\right)$ in some relation of $S$, where $k>1$, and at least two vertices in $\bar{x}$ are distinct; say these two vertices are $x_{i}$ and $x_{j}$ (this is possible as $S$ is non-null). Consider the substructure $X$ of $S$ induced by the vertices in $\bar{x}$. As the age of $S$ contains $X$, is closed under isomorphism, and has free amalgamation, the age of $S$ contains the structure $B^{\prime}$ formed by the union of two isomorphic copies of $X$ with intersection $\left\{x_{i}\right\}$. Label the two distinct copies of $x_{j}$ in $B^{\prime}$ as $x_{j 1}$ and $x_{j 2}$. As the minimum arity of a relation is at least 2, there is exactly one isomorphism type of structure in the age of $S$ with one vertex. Hence, we identify $x_{j 1}$ and $x_{j 2}$ with $u$ and $v$, respectively. See Figure 2 .

Let $A_{1}$ be the substructure of $S$ induced by $\{u, v\}$, and let $A$ be the substructure of $S$ induced by $\{u, v\} \cup T$. Let $B$ be the union of $B^{\prime}$ and $A$ over $A_{1}$. As $S$ is homogeneous, we may amalgamate $B$ into $S$ over $A$. Hence, there is a vertex $z \in V(S)$ (corresponding to the isomorphic image of $x_{i}$ ) joined in $G(S)$ to both $u$ and $v$ but not $T$.

Not all relational structures with distinguishing number 2 are weake.c. (for example, consider the infinite binary tree). An open problem is to determine a necessary and sufficient condition for a countably infinite relational structure to have distinguishing number 2.

\section{References}

[1] M. O. Albertson, K. L. Collins, Symmetry breaking in graphs, The Electronic Journal of Combinatorics 3 (1996) \#R18. MR1394549

[2] A. Bonato, D. Delić, On a problem of Cameron's on inexhaustible graphs, Combinatorica 24 (2004) 35-51. MR2057682 
[3] P. J. Cameron, The random graph, In: The Mathematics of Paul Erdös, II, Algorithms and Combinatorics, 14, Springer, Berlin, 1997, pp. 333351. MR1425227

[4] R. Fraïssé, Theory of relations, Revised edition, with an appendix by Norbert Sauer, North-Holland Publishing Co., Amsterdam, 2000. MR1808172

[5] W. Imrich, S. Klavžar, V. Trofimov, Distinguishing infinite graphs, The Electronic Journal of Combinatorics 14 (2007) \#R36. MR2302543

[6] A. H. Lachlan, R. E. Woodrow, Countable ultrahomogeneous undirected graphs, Trans. Amer. Math. Soc. 262 (1980) no. 1, 51-94. MR0583847

[7] C. Laflamme, L. Nguyen Van Thé, N.W. Sauer, Distinguishing number of countable homogeneous relational structures, submitted.

[8] M. E. Watkins, X. Zhou, Distinguishability of locally finite trees, The Electronic Journal of Combinatorics 14 (2007) \#R29. MR2302536

AnTHONY Bonato

Department OF MATHEMATiCs

RYERSON UNIVERSITY

TORONTO, ON

CANADA

E-mail address: abonato@ryerson.ca

DeJAN Delić

Department OF MATHEMATiCs

RYERSON UNIVERSITY

TORONTO, ON

CANAdA

E-mail address: ddelic@ryerson.ca

Received March 29, 2010 